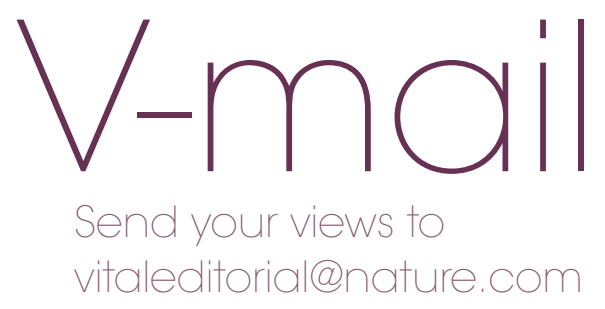

\section{A JOB NOT A PROFESSION}

I have just read the letters in the autumn issue of Vital regarding the changes in our roles (page 4).

I work for the primary dental service which is part of the NHS system. We as a service deal with patients who are not normally seen in practice due to mental or physical problems. Our patients can be violent and being kicked is seen as part of the job. We are shouted at by some of the patients; again this is taken by the staff as part of the behaviour we can expect. For this we are treated the same as dental nurses in general practice and our pay is the same. We have not had pay increases like other NHS staff. We also do domiciliary visits and are expected to drive to these places and attend our clinics within the service. Our employers this year have insisted that to claim mileage or parking back we have to change our private car insurance; many of us have had to increase this by $£ 30$ or more. Again we have the indemnity as well as GDC registration costs to find. We have also been told these costs may increase due to the type of patients we treat.

I think that at present a large unplanned shortfall of dental nurses is developing across both the private and NHS sectors. As we now register within our own rights we need to take out expensive new policies but due to poor pay in the past our employers are not necessarily increasing wages to allow dental nurses to fulfil their obligations.

Due to the fact I do work for the NHS the only perk we do get is that we have to attend so many training sessions and staff meetings, so collecting CPD hours is not a problem. I feel very sorry for the dental nurse who needs to gain hours in practice.

Many of the new guidelines that have been brought out for the safety of patients and staff appear to be over the top with a number of them 'forgotten' or 'stopped' unless inspections are being carried out. Many of the new rules for HTM 01-05 are very costly and time consuming and thus are forgotten in the never-ending race to get the surgery ready for the next patient.

When I started dental nursing we tried to win patients over that dentists were not there for a 'fast buck', and showed that we cared about them and wanted to do the best we could. However, due to the pressures on dental nurses our jobs have changed to having little or no time for the patients and their concerns. We are able do very little to change the next generation's perceptions on dentistry being painful and indeed, out for a quick buck.

I know a number of very experienced nurses leaving the profession as they are no longer gaining pleasure from treating patients and financial rewards are lacking. If dental nurses do not start to be treated better, practice principals will find it hard to maintain their practices as they are being run at the moment. Many of the 'old style' nurses are prepared to work through lunch etc for the benefit of the patient but the new nurses have a different outlook. I wish I was one of them and didn't worry or get as stressed if I don't do a great job. I am looking for a new job as I feel this is a job rather than a profession to be proud of.

\section{Name and address supplied}

\section{UNINTENDED CONSEQUENCES}

The GDC's requirement for all dental nurses to be either qualified or on a training programme is well intentioned but is having unintended consequences.

Local colleagues and I have employed nurses with recent qualifications from a local training college only to find they have little knowledge of how to handle dental materials and are unable to do even basic charting. Without this latter skill they risk the wrong treatment being done to a patient and they are therefore unsafe working in a practice despite them possessing a qualification approved by GDC, whose principal role is the protection of the public.

In contrast, I recently needed to employ a part-time dental nurse, a position which would be ideal for a lady who used to be a dental nurse before raising a family and now wishes to do a few days' work. Such ladies are normally highly dependable as well as having a breadth of life experiences which count for so much when interacting within a team and with the patients. Several such ladies would have been ideal but they were unable to consider the post as they have no formal qualifications and it is unreasonable to require them to undertake a 'recognised training programme', at extra cost and in their own family time, for something which they can do standing on their head. Additionally, each of us GDPs works in our own quirky way and finding the right nurse can be a fine art.

The GDC's well-intentioned rules are therefore forcing a whole group of potential team members to be barred from consideration. This can surely not have been the intention of the GDC and so I propose an alteration to the rules: dental nurses who have had several years of experience before taking a career break should be allowed to return to work under the supervision of a nominated dentist; that dentist would then be entirely accountable for the actions of that nurse; there could also be a form of in-house assessment which could gain recognition from the GDC.

By adopting this scheme, the profession would welcome back a whole group of eminently suitable (but not officially qualified) nurses, dentists would be able to work with a nurse who matches their quirky ways, and the protection of the public (the principal role of the GDC) would be enhanced by having nurses who can accurately chart.

\section{Marks}

This letter was originally published in the BDJ

(2013; 213: 264-265). 